\title{
Une jambe de trop
}

\section{Erhard Taverna}

Dr méd., membre de la rédaction

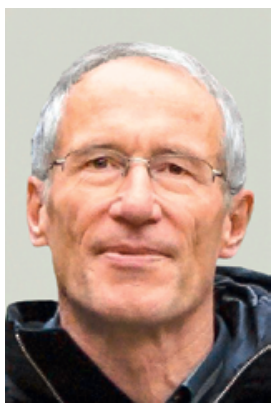

Il s'appelait John. Un homme vigoureux qui, en entrant dans le cabinet, portait toujours deux doigts à son stetson. Un diabétique avec un moignon de jambe impeccable, qu'il déballait presque amoureusement en salle de consultation. Un tragique accident de voiture aux Philippines avait nécessité l'amputation au-dessus du genou gauche. C'était la version officielle avancée par le grand voyageur et ingénieur demandé. Il le gérait étonnamment bien et semblait en paix avec lui-même et avec le monde. Lorsque les questions finissaient par l'importuner, il avait l'habitude de citer Matthieu: «Si ta main ou ton pied est pour toi une occasion de chute, coupe-les et jette-les loin de toi». Ce que les gens prenaient pour une blague cynique était en fait très proche de la vérité. John s'était volontairement et à grands frais fait amputer de sa jambe en bonne santé. Si nous n'avons aucun mal avec les situations où existe une contrainte extérieure visible, les situations de détresse intérieure à l'origine d'actes de mutilation défient souvent notre compréhension. Il en va ainsi des personnes atteintes d'un trouble identitaire de l'intégrité corporelle (TIIC). Si beaucoup d'aspects restent obscurs, un terme technique médical calme un peu les esprits. Ce sont en priorité des hommes disposant d'une bonne formation qui se rendent en ExtrêmeOrient pour se faire amputer d'une jambe (le plus souvent la gauche) en bonne santé. Selon les forums d'entraide sur Internet, le désir d'amputation, d'aveuglement ou même de paraplégie n'est pas si rare. Dans le jargon technique, cela s'appelle la xénomélie, le sentiment qu'un membre ne nous appartient pas. Les personnes concernées ne se démarquent pas par des particularités psychiatriques, mais se plaignent dès avant la puberté d'une perception pénible de leur corps. Il existe de nombreuses autres notions décrivant d'autres syndromes liés à un défaut d'intégrité entre le corps et le soi. Le plus répandu est l'absence d'identité sexuelle, connu sous le nom de trouble de l'identité de genre, pour lequel les traitements chirurgicaux et hormonaux sont légaux et au moins en partie pris en charge par les caisses-maladie. On établit ici une distinction avec l'apotemnophilie, qui correspond à une excitation sexuelle liée à une amputation réelle ou imaginaire, et avec la misoplégie, qui est une aversion pour une partie de son corps pouvant aller, dans les cas extrêmes, jusqu'au syndrome de Cotard, où le sujet se croit mort. C'est en 1880 que Cotard, un neurologue français, a pour la première fois décrit ce tableau clinique qui peut survenir chez les schizophrènes, les personnes atteintes d'une dépression sévère ou qui ont subi des lésions à la suite d'un AVC, d'une épilepsie ou d'une tumeur cérébrale. Le point commun de ces patients atteints de xénomélie, ce sont des altérations visibles par TEP ou magnétoencéphalographie (MEG) de l'activité cérébrale au niveau du lobe frontal et pariétal, ainsi que du cortex prémoteur. Comme ces réseaux sont généralement latéralisés dans l'hémisphère droit du cerveau, les désirs d'amputation concernent des parties situées du côté gauche du corps. Les particularités anatomiques qui, conjointement à des facteurs génétiques et sociaux, laissent supposer des origines complexes, ne permettent pour l'instant pas de traitement des causes, hormis, en dernier recours, l'amputation. Les succès sont éloquents. Selon une en quête réalisée auprès de 21 personnes concernées âgées de 23 à 73 ans, toutes étaient plus satisfaites après une opération réussie à l'étranger. Certaines avaient des difficultés à gérer leur nouvelle vie quotidienne, mais aucune ne regrettait sa décision.

Les médecins sont-ils autorisés à retirer des parties du corps en bonne santé lorsque la souffrance des patients diminue nettement et peut-être à vie après une amputation réussie? La psychothérapie ou les médicaments sont généralement inefficaces. Une personne qui veut se débarrasser de sa jambe finira par recourir à la tronçonneuse, se blesser avec de la neige carbonique ou garrotter le membre concerné. Si la transsexualité avec ou sans suivi médical est largement acceptée, la suppression de parties du corps superflues en lien avec une représentation corporelle défaillante reste contestée. Une association pour la promotion des études sur les troubles identitaires souhaite y remédier, des forums en ligne mettent en relation les personnes atteintes de TIIC et leurs proches. Même si les estimations concernant la fréquence de ces troubles divergent fortement, le débat sur le droit à l'automutilation a gagné la Suisse depuis un bon moment.

\section{Références}

- The desire for healthy limb amputation - structural brain correlates and clinical features of xenomelia. Brain 2012, 3 octobre, Prof. Brugger, USZ.

www.forum.biid.ch www.vfsk.eu 